\title{
On the Use of 3dB Qualification Margin for Structural Parts on Expendable Launch Vehicles
}

\author{
Isam Yunis ${ }^{1}$ \\ NASA Langley Research Center, Hampton, VA 23681 \\ Damian Ludwiczak ${ }^{2}$ \\ NASA Glenn Research Center, Cleveland, OH 44135
}

\begin{abstract}
The standard random vibration qualification test used for Expendable Launch Vehicle components is Maximum Predicted Environment (MPE) + 6dB for a duration of 4 times the service life of the part. This can be a severe qualification test for these fatigue-sensitive structures. This paper uses flight data from several launch vehicles to establish that reducing the qualification approach to MPE $+3 \mathrm{~dB}$ for the duration of the peak environment (1x life) is valid for fatigue-sensitive structural components. Items that can be classified as fatigue-sensitive are probes, ducts, tubing, bellows, hoses, and any non-functional structure. Non-functional structure may be flight critical or carry fluid, but it cannot include any moving parts or electronics. This reduced qualification approach does not include primary or secondary structure which would be exclusively designed by peak loads, either transient or quasi-static, that are so large and of so few cycles as to make fatigue a moot point.
\end{abstract}

\section{Introduction}

$\mathrm{T}$ he standard qualification approach for random vibration used for Expendable Launch Vehicle (ELV) components is Maximum Predicted Environment (MPE) $+6 \mathrm{~dB}$ for 4 times the service life of the part ${ }^{1}$. MPE is most often defined as the P95/50 level, where P95/50 is the statistical level which will be greater than $95 \%$ of the spectral peaks with $50 \%$ confidence.

The service life of a component includes all vibration environments including stress screening, acceptance testing, and flight. The duration of acceptance testing is often unknown since many tests may need to be run. For example, if an internal part fails during an acceptance test, the faulty part is replaced and the acceptance test is rerun, thereby subjecting the rest of the items to another exposure. Because of the uncertainty in the acceptance duration, many programs choose to use a qualification test duration of 180 seconds for all items. This duration typically covers $4-6$ acceptance tests.

The above approach (MPE $+6 \mathrm{~dB}$ for $4 \mathrm{x}$ life) demonstrates peak load margin and fatigue margin over known environments. For complex electronics or mechanical components, this testing may be the only vibration evaluation because analysis may be infeasible and failure modes may be hard to characterize. Because of this, the robust qualification approach also has the advantage of providing insurance against unknowns. Most significantly, though, this methodology has proven successful throughout ELV history. This success cannot be quickly discarded.

For fatigue-sensitive structural (non-operational) components, MPE $+6 \mathrm{~dB}$ can be a very severe environment. After all, $6 \mathrm{~dB}$ is a factor of 4 increase on the magnitude of the Power Spectral Density (PSD) and a factor of 2 increase on the acceleration. Typical structural qualification tests use factors of 1.2-1.42. So a factor of 2.0 is quite high. This paper establishes MPE (P95/50) $+3 \mathrm{~dB}$ for peak event duration (1x life) as an acceptable and reasonable qualification approach for non-operational structural items.

Components that fall under the classification of fatigue-sensitive are probes, ducts, tubing, bellows, hoses, and any non-functional structure. Non-functional structure may be flight critical or carry fluid, but it cannot include any moving parts. This does not include primary or secondary structure which would be exclusively designed by peak loads, either transient or quasi-static, that are so large and of so few cycles as to make fatigue a moot point.

\footnotetext{
${ }_{1}^{1}$ Aerospace Engineer, Structural and Thermal Systems Branch, MS 431, AIAA Senior Member

${ }^{2}$ Aerospace Engineer, Structural Systems Dynamics Branch, MS 86-10 


\section{The Standard 6 dB Approach}

Before beginning a discussion of qualification, it is important to set the technical basis of the discussion.

\section{A. Structural Fatigue Equivalence}

In the case of random vibration, a useful approach to address fatigue is the Miles-Miner hypothesis ${ }^{3}$, where it is assumed:

1. $\mathrm{N} \sigma^{\mathrm{b}}=\mathrm{C}$ is a valid expression of a structural material S-N data.

Where: $\quad \mathrm{N}=$ Number of cycles to failure at stress $\sigma$

$\mathrm{b}=$ Exponent for the material

$\mathrm{C}=$ Constant for the material

2. The random vibration excites the system to resonance. The system response is narrow band random, where the assumption of the Rayleigh peak distribution is valid.

3. Peaks of $3 \sigma$ do not exceed the yield strength of the material.

Miner's hypothesis is represented by Eq. 1:

$$
D_{r}=\sum_{i=1}^{T} \frac{n_{i}}{N_{i}}
$$

Where:

$D_{r}=$ damage ratio due to random input; when $D_{r} \geq 1$, failure occurs

$\mathrm{n}_{\mathrm{i}}=$ number of completely reversed stress cycles at $\sigma_{\mathrm{i}}$ (cycles)

$\mathrm{N}_{\mathrm{i}}=$ number of cycles to failure at $\sigma_{\mathrm{i}}$ (cycles)

$\mathrm{n} \quad=$ number of different stress levels $\sigma_{\mathrm{i}}$

If $t_{i}$ is the time duration (in seconds) at $\sigma_{i}$ and $F_{i}$ is the frequency $(\mathrm{Hz})$ of the structural response at which $\sigma_{i}$ oscillates, then the variable $\mathrm{n}_{\mathrm{i}}$ can be expressed directly as:

$$
n_{i}=t_{i} F_{i}
$$

Since $\mathrm{N} \sigma^{\mathrm{b}}=$ C, Eq. 1 can then be transformed to:

$$
D_{r}=\sum_{i=1}^{n} \frac{\sigma_{i}^{b} t_{i} F_{i}}{C}
$$

The values of $\sigma_{i}$ and $F_{i}$ will be a function of the structure under test. The root mean square (RMS) stress $(\sigma)$ for a structure at a particular modal frequency can be estimated from Miles' equation ${ }^{4}$ :

$$
\sigma_{i}=K_{i} \sqrt{\frac{\pi P_{i} F_{i} Q_{i}}{2}}
$$

Where:

$\mathrm{K}_{\mathrm{i}}=$ factor relating stress to acceleration at frequency $\mathrm{F}_{\mathrm{ni}}(\mathrm{psi} / \mathrm{g})$

$\mathrm{P}_{\mathrm{i}}=$ value of PSD spectrum at $\mathrm{F}_{\mathrm{ni}}\left(\mathrm{g}^{2} / \mathrm{Hz}\right)$

$\mathrm{F}_{\mathrm{i}}=$ frequency of structural mode $\mathrm{i}(\mathrm{Hz}=$ cycles $/ \mathrm{sec})$

$\mathrm{Q}_{\mathrm{i}}=$ quality factor for structural mode $\mathrm{i}=1 /(2 \zeta)$ 
Eqs. 3 and 4 can be combined to define the structural damage estimate due to a random vibration environment:

$$
D_{r}=\sum_{i=1}^{n} \frac{\left(K_{i} \sqrt{\frac{\pi P_{i} F_{n i} Q_{i}}{2}}\right)^{b} t_{i} F_{n i}}{C}
$$

Equation 5 has known issues, such as the fact that it uses the RMS stress rather than the peak stress, thereby under predicting the damage. However, Eq. 5 is not the end product. Rather than an absolute value of the damage, we desire an equation for equivalent damage, which is the ratio of two different damage ratios $\left(\mathrm{D}_{\mathrm{r} 2} / \mathrm{D}_{\mathrm{r} 1}\right)$. This will be the fatigue equivalence equation for structural testing.

In Eq. 5 there are only two variables that differ between flight and the qualification vibration test. These are the input level $\mathrm{P}_{\mathrm{i}}$ and the duration $\mathrm{t}_{\mathrm{i}}$. The change in the amount of structural damage based on the values of these two variables can be calculated from Eq. 5 as:

$$
\frac{D_{r 2}}{D_{r 1}}=\left(\frac{P_{2}}{P_{1}}\right)^{\frac{b}{2}} \frac{t_{2}}{t_{1}}
$$

For fatigue equivalence, the ratio $D_{\mathrm{r} 2} / \mathrm{D}_{\mathrm{r} 1}=1$, such that:

$$
\frac{t_{2}}{t_{1}}=\left(\frac{P_{1}}{P_{2}}\right)^{\frac{b}{2}}
$$

Equation 7 is the fatigue equivalence equation. Consider two sources of damage for a flight article: Acceptance testing and flight. (If stress screening is also conducted, this can easily be added to the argument.) Let the subscript "a" refer to "Acceptance Testing", the subscript " $\mathrm{f}$ " refer to "flight", the subscript "q" refer to qualification testing, and the subscript " 1 " refer to qualification for one fatigue life. The qualification test time is written as:

$$
t_{q 1}=\left(\frac{P_{a}}{P_{q}}\right)^{\frac{b}{2}} t_{a}+\left(\frac{P_{f}}{P_{q}}\right)^{\frac{b}{2}} t_{f}
$$

Typically, $\mathrm{P}_{\mathrm{a}}=\mathrm{P}_{\mathrm{f}}$ because the acceptance level is the flight level. This is not true if the flight levels are so low that workmanship considerations outweigh vibration qualification. For the purposes of this discussion, we assume $P_{a}=P_{f}$ as this is the most common and most critical condition. Even when $\mathrm{P}_{\mathrm{a}}=\mathrm{P}_{\mathrm{f}}$, the right side of Eq. 8 cannot be collapsed into a single term. This is because $\mathrm{P}_{\mathrm{f}}$ is a statistical term, typically P95/50. While acceptance testing on a shaker is controlled, the actual flight environment is unknown until flight. Therefore, margin must be placed on the flight environment but not on acceptance testing. For simplicity it is assumed that the worst possible flight environment which will be considered is the qualification environment, such that $\mathrm{P}_{\mathrm{f}}=\mathrm{P}_{\mathrm{q}}$. Any value can be chosen, but this approach allows the test magnitude to be the maximum level ever expected in flight, even though this value will probably never occur. With this assumption, Eq. 8 becomes:

$$
t_{q 1}=\left(\frac{P_{a}}{P_{q}}\right)^{\frac{b}{2}} t_{a}+t_{f}
$$

The fatigue equivalence is shown in Eq. 9. However, fatigue margin must be shown. Fatigue scatter factors are applied to Eq. 9 to develop the qualification duration. Per Ref. 1, a fatigue scatter factor of 4 is appropriate. Applying this factor leads to the final qualification duration equation consistent with Ref. 1: 


$$
t_{q}=4\left[\left(\frac{P_{a}}{P_{q}}\right)^{\frac{b}{2}} t_{a}+t_{f}\right]
$$

In Eq. 10, the S-N curve variable $\mathrm{b}$ is obtained from the material characteristics of the structure being analyzed for damage. We note that $b$ is an exponent which can have a rather large effect on the value of $t_{q}$ being calculated. Different materials will require different $b$ values. However, most qualification vibration testing is for electronics boxes or mechanical parts. In this case, many materials are involved as well as mechanical wear. For this condition, a value of $b=4$ is recommended ${ }^{5}$. Since $\mathrm{P}_{\mathrm{a}} / \mathrm{P}_{\mathrm{q}}<1$, using $\mathrm{b}=4$ should be conservative for the vast majority of cases as most metals have values of $\mathrm{b}$ between 6 and 9 .

\section{B. Uncertainty in Application}

Although, the preceding discussion appears rigorous and clear, the application of vibration qualification actually involves a significant amount of uncertainty. The following points illustrate the difficulty in any discussion of vibration qualification:

- The quality of the numbers used for the derivation of the vibration environment (MPE) is often quite poor. The data comes from either flight or ground test. Flight data suffers from quantity, as there are typically very few data points for the statistical derivation. Test data suffers from quality, as the flight conditions are never duplicated accurately in any test.

- Existing data is often extrapolated to different flight conditions, further weakening the validity of use.

- Statistics are applied to the available data. Here, historical assumptions about the expected distribution of the data are used. Log-normal is the standard assumption ${ }^{5}$. Also, various methods are used to arrive at the statistical mean $\left(\mu_{\mathrm{x}}\right)$ and standard deviation $\left(\sigma_{\mathrm{x}}\right)$ given a small sample size. So, not only is the data limited, the processing of the data introduces further uncertainty.

- Statistics are applied assuming stationary data, when ELV flight data is actually non-stationary. The typical random vibration environment is defined as maxi-max, which is a level computed as the maximum of 1 second PSD's across the event. With this method, frequency domain peaks from different times become part of a composite PSD. During testing, then, this 1 second peak is considered to occur across the duration of the event. The duration of the event is considered to be the time at which the levels are within $6 \mathrm{~dB}$ of the peak [Ref. 5]. This approach is obviously conservative but this conservatism is not quantified.

- Failure modes and failure limits are often not well understood for "black boxes". Black boxes include electronic items, functional items (relays), or any other item for which the failure modes are complex or numerous or undefined. The choice of $b=4$ is assumed to cover all failure modes.

- For many black boxes, manufacturing repeatability must be considered in qualification. Poor repeatability calls into question the entire qualification as failure modes can change dramatically. Even with good repeatability, there will be some variability from unit to unit. This variability may diminish the assumed margin of the test.

- Test fixtures and mounting conditions do not duplicate flight boundary conditions. This fact results in test responses that may or may not approximate the flight responses.

- Tests are often performed in one axis at a time. Some failure modes may be a function of multi-axis vibration.

These fundamental issues would seem to completely invalidate the vibration qualification process. However, in spite of these issues, historical flight success shows that this methodology works. Therefore, abandoning the historical practice should not be taken lightly. None-the-less, the uncertainties certainly show that there is room for modification. In fact, Section III below shows that modification of the standard approach is valid for simple fatiguesensitive structures.

\section{Fatigue-sensitive Structures}

Fatigue-sensitive structures are those which are non-functional in flight (such as a probe used just during tanking operations) and those without any moving parts (such as ducts, tubing, bellows, and hoses). They may be flight critical or carry fluid and it may be flexible, but it cannot include any moving parts. Fatigue-sensitive structure does 
not include primary or secondary structure which would be exclusively designed by peak loads, either transient or quasi-static, that are so large and of so few cycles as to make fatigue a moot point.

Fatigue-sensitive structures can be distinguished from the generic $(\mathrm{MPE}+6 \mathrm{~dB})$ case for several reasons. Fatiguesensitive structure does not suffer from the black box issue of unknown failure modes. Because of this, analysis is often possible to add confidence beyond testing. Further, manufacturing repeatability is much more reliable for structure than it is for black-boxes. Because of this, fatigue-sensitive structures rarely undergo acceptance testing. These significant factors make fatigue-sensitive structures candidates for reduced margin.

\section{The $3 \mathrm{~dB}$ Approach}

In order to address reduced qualification approaches for fatigue-sensitive structure, a study of launch vehicle data was conducted in order to quantify the degree of conservatism in the standard approach. Measurements from tip to tail were considered in three vehicle configurations (Figs. 1-3). For proprietary reasons the vehicles cannot be identified. Locations near the engine have a consistent vibration environment during flight. Locations nearer the payload have vibration environments that vary throughout the flight. The "Test Spec" shown in these figures is a random vibration time history based on an MPE PSD derived using the classical maxi-max approach; this represents a time history that a shaker table may produce. No margin has been added to the "Test Spec" time history; it is merely the maxi-max approach that causes the large values.

The "fatigue" due to the complete flight data was compared to the "fatigue" due to applying the MPE and $\mathrm{MPE}+3 \mathrm{~dB}$ for the event duration ( $1 \mathrm{x}$ life). These results are shown in Table 1. The "fatigue" was computed by treating the flight data as structural stress data and summing the stress cycles using the theory of Section II. The event duration is considered to be the duration of vibration within $6 \mathrm{~dB}$ of the peak. The results show that even testing for short events covered the life of the flight. With $3 \mathrm{~dB}$ margin, a minimum of 4 lives was addressed, with most values greater than 10 lives. While these examples are not inclusive of the full spectrum of possible flight data, they are representative with conclusive results. Fatigue of fatigue-sensitive structure is not an issue for launch vehicles if current MPE development processes are used and test levels are at least MPE+3dB for one life.

The issue of peak loads is a more difficult issue to discuss for statistical environments like random vibration. However, the same study that defined Table 1 was used to investigate the expected peak loads from the MPE versus that from the flight data. The results are shown in Table 2. As expected, using the MPE levels creates a marginally conservative structural response due to the maxi-max approach. Adding $3 \mathrm{~dB}$ of test margin adds a factor of 1.4 to the test levels, which is greater than the typical metallic safety factors [Ref. 2].

The time signal from a PSD is not a valid comparison mechanism for peak structural response as the time signal is random and the peak values will be random. Also, comparisons of peak load using statistical quantities like RMS can be misleading because frequency content is lost. Therefore, for this study, the shock response spectrum (SRS) was used. The SRS's for the flight data and for a time signal derived from the MPE+3dB PSD are shown in Figures $1 \mathrm{~b}, 2 \mathrm{~b}$, and $3 \mathrm{~b}$. The results in the plots show that there is at least a factor of 1.5 in the MPE+3dB test, which is greater than the typical metallic safety factors. Therefore, the use of MPE+3dB for the peak environment duration (1x life) structural items creates a low risk due to reduced margin on peak loads.

In the above discussion and in Tables 1 and 2, a minimum "life" of 10 seconds was considered. This was done because the conservative development of the MPE is lost at very short durations and because a very short shaker test is not realistic. Therefore, all conclusions here are based on a 10 second minimum life. A higher minimum time would result in more conservative results. In fact, the results in Table 1 clearly show a correlation between event duration and conservatism. This result is due to the maxi-max approach, which envelopes peak environments from each second of data. It is easy to see that the use of the maxi-max approach is not a good idea for stationary data.

Finally, this study shows the applicability of $\mathrm{MPE}+3 \mathrm{~dB}$ for qualification of structure without an important consideration: The MPE is typically defined as a smooth envelope of the PSD. Enveloping can add a very large amount of conservatism into the test process. The enveloping factor was not considered, but adds even more confidence to the conclusions drawn above.

This assessment seems to make an argument for MPE $+3 \mathrm{~dB}$ as a new standard for all items. This is not so for the following reasons:

- Structures have a manufacturing repeatability that is not seen in black boxes. Furthermore, structures have inspection processes to control the repeatability; black boxes do not. The above study shows that MPE $+3 \mathrm{~dB}$ is sufficient for fatigue scatter, but that MPE $+3 \mathrm{~dB}$ has no additional margin for manufacturing variability.

- Structures have known linear failure modes. Black boxes may have unknown failure modes. These failure modes may be due to non-linear interactions, wear, relay chatter, or poorly understood connectivity. These failure modes do not follow the fatigue rules. Because of this, the above assessment does not apply. 
- If the statistics are to be believed, MPE $+3 \mathrm{~dB}$ will be exceeded from time to time. Because of the known failure modes of structure and the manufacturing repeatability, the tables above show margin against small exceedences. A black box has no margin beyond its tested condition because of the unknown failure modes that have not been quantified.

The use of MPE $+3 \mathrm{~dB}$ does not apply to the qualification of primary or secondary structure. A random vibration test for these structures does not make sense, but if one is used, the levels should be much higher and consistent with structural testing. For example, when limit load is defined as $3 \sigma$ or P99/90, margin should be added to that. This may mean a test at P95/50 $+9 \mathrm{~dB}$. Again, this is not an approach advocated for qualification of primary or secondary structure.

\section{Conclusion}

Launch vehicle flight data has been processed to show that random vibration qualification to MPE $+3 \mathrm{~dB}$ for peak event duration ( $1 \mathrm{x}$ life) is a valid qualification approach for fatigue-sensitive structural components. It has been shown that current specification development techniques, particularly the maxi-max approach, allow tests for the event duration ( $1 \mathrm{x}$ life) to actually qualify the structure for 4 lives. The data also shows that testing to MPE $+3 \mathrm{~dB}$ for the event duration provides the required amplitude margin.

\section{Acknowledgments}

This work was completed for the NASA Launch Services Program at the Kennedy Space Center.

\section{References}

1 “Test Requirements for Launch, Upper-Stage, and Space Vehicles,” MIL-STD-1540C, 15 September, 1994.

2 "Structural Design and Test Factors of Safety for Spaceflight Hardware," NASA Technical Standard 5001, June 21, 1996.

3 Miner, M.A., "Cumulative Damage in Fatigue," Journal of Applied Mechanics, Vol. 12, Transactions of the ASME, Vol. 67, pp. A159-A164.

4 Miles, John W., “On Structural Fatigue Under Random Vibration Loading,” Journal of Aeronautical Sciences, p. 753, November, 1954.

5 ”Dynamic Environmental Criteria,” NASA Technical Handbook, NASA-HDBK-7005, March 13, 2001. 

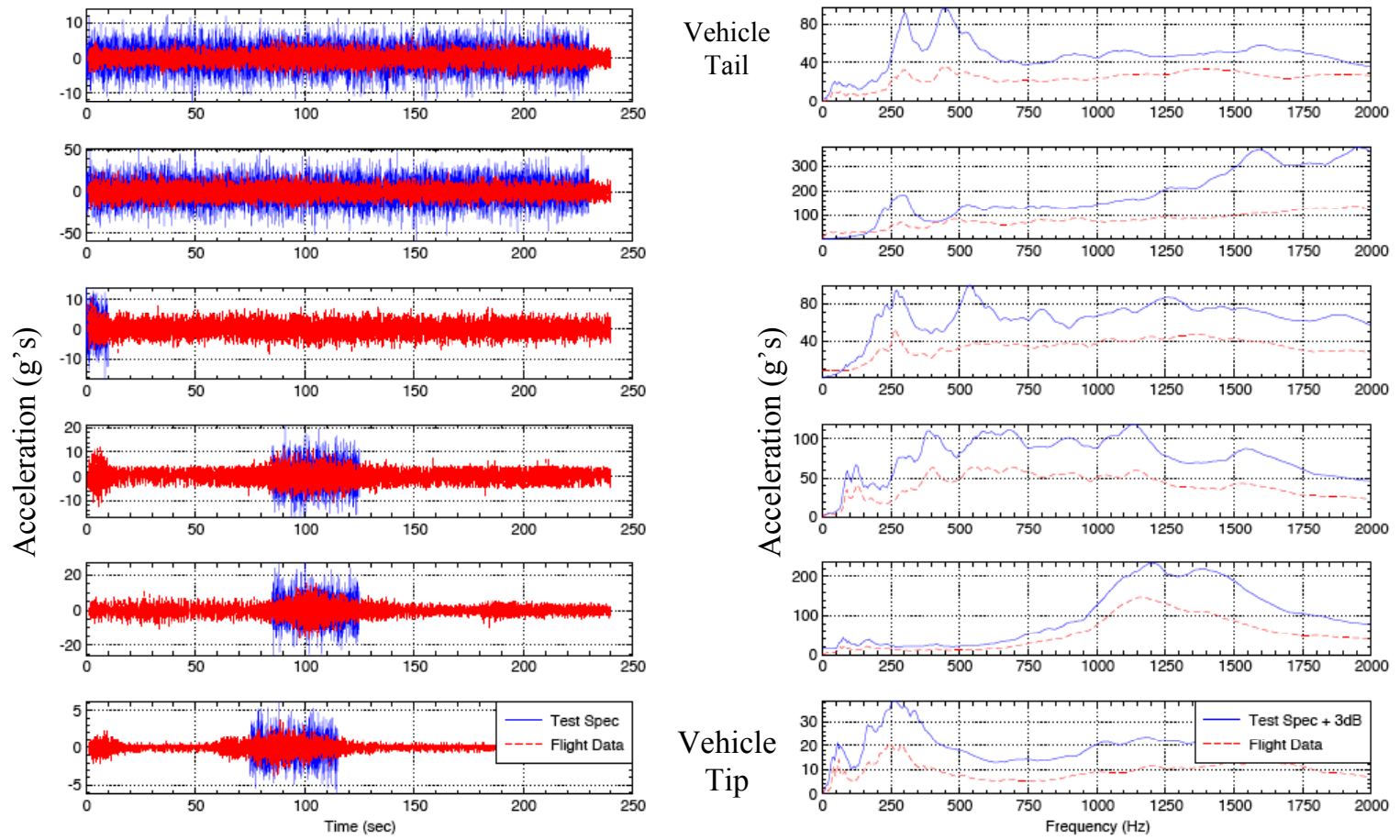

(a)

(b)

Figure 1. Data and derived test time histories from Launch Vehicle \#1. Acceleration flight data from various stations along the Launch Vehicle from aft to forward (moving down the page) is shown over the maxi-max derived environment in (a). The Shock Response Spectra (SRS's) of the two signals are shown in (b).
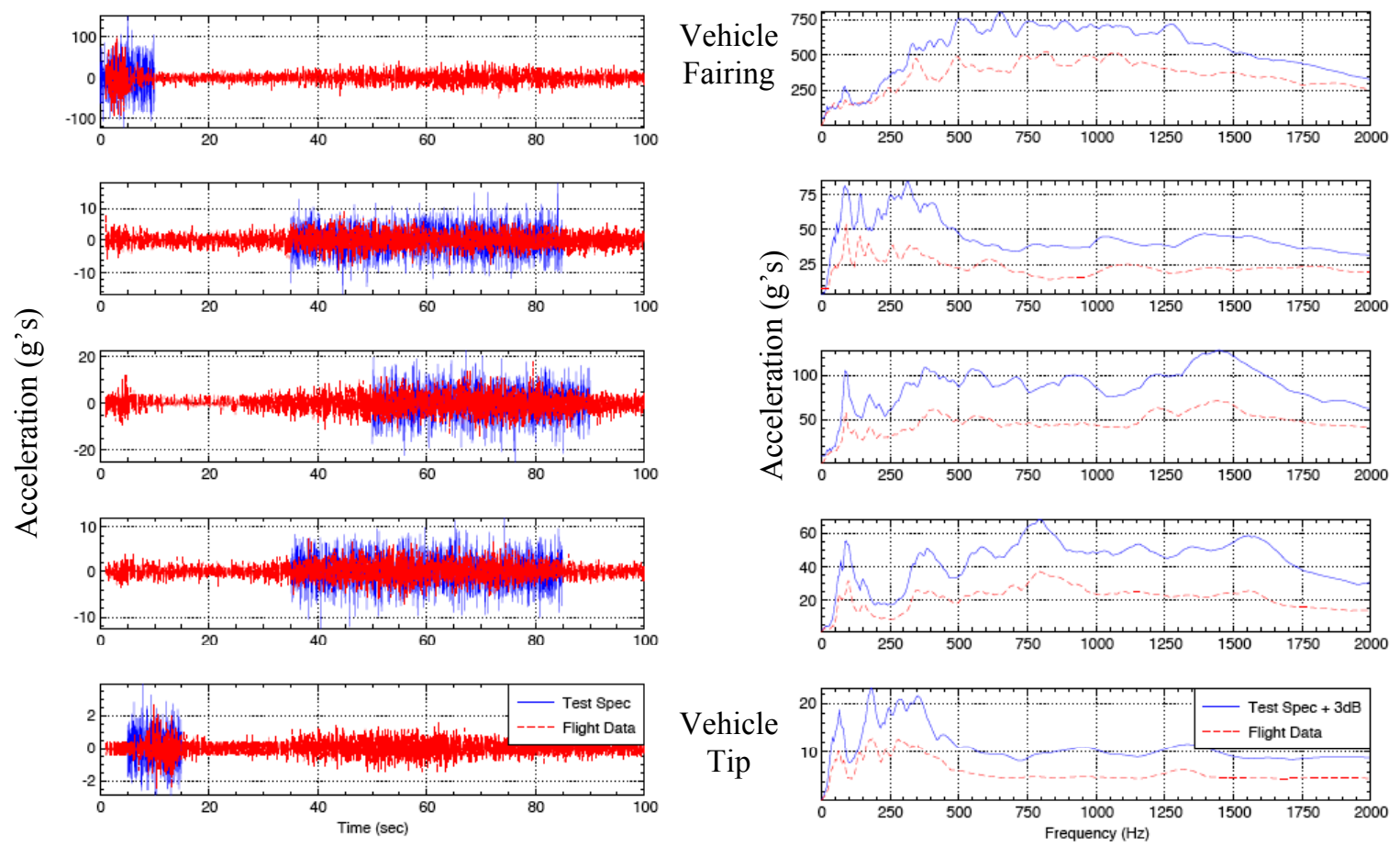

(a)

(b)

Figure 2. Data and derived test time histories from Launch Vehicle \#2. Acceleration flight data from various stations along the Launch Vehicle from aft to forward (moving down the page) is shown over the maxi-max derived environment in (a). The Shock Response Spectra (SRS's) of the two signals are shown in (b). 

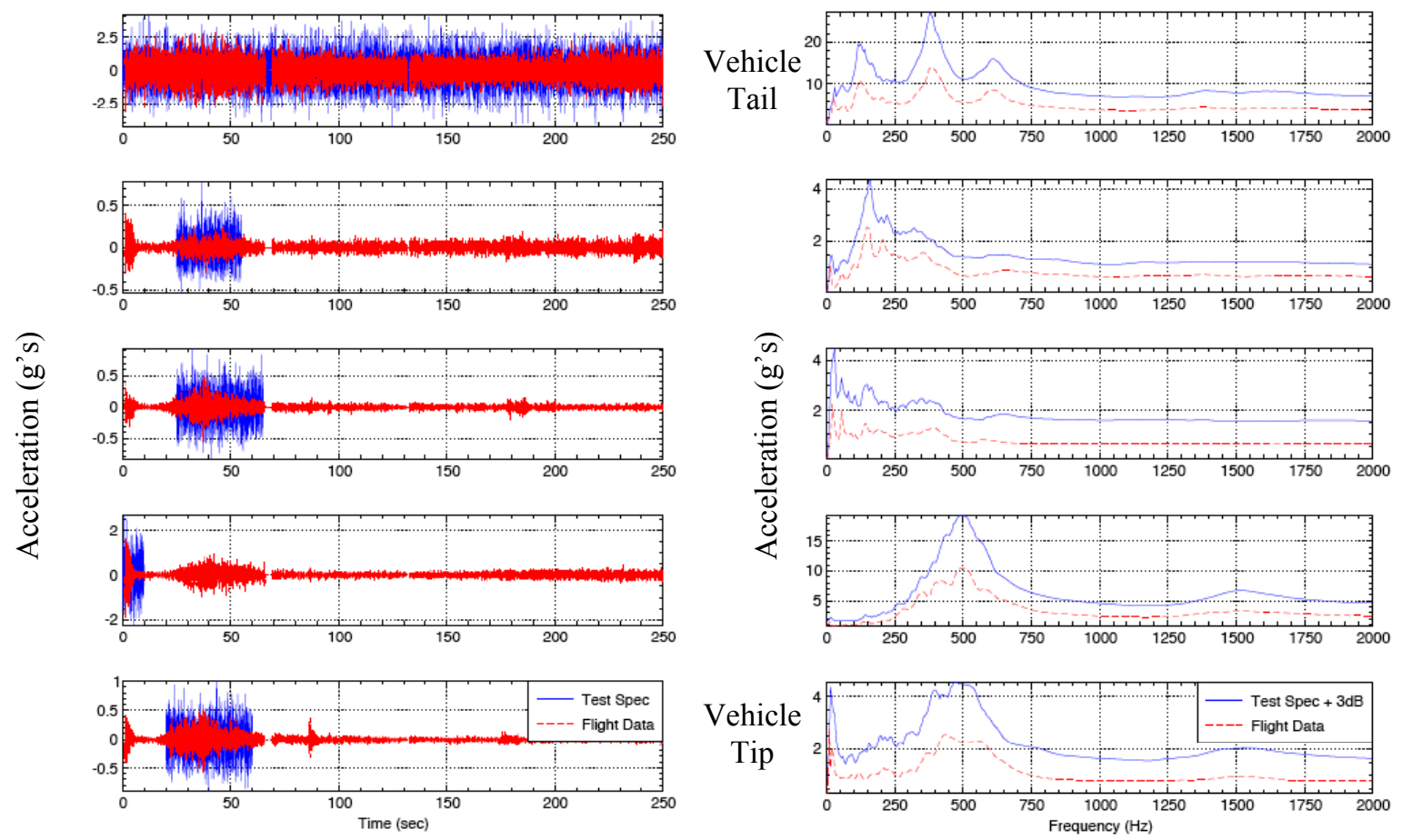

(a)

(b)

Figure 3. Data and derived test time histories from Launch Vehicle \#3. Acceleration flight data from various stations along the Launch Vehicle from aft to forward (moving down the page) is shown over the maxi-max derived environment in (a). The Shock Response Spectra (SRS's) of the two signals are shown in (b). 
Table 1. Comparison of Fatigue Life Expended as Computed Using ELV Flight Data, the Associated MPE, and the Associated MPE+3dB.

\begin{tabular}{|c|c|c|c|c|c|c|c|c|c|}
\hline & \multicolumn{3}{|c|}{ Configuration 1 } & \multicolumn{3}{c|}{ Configuration 2 } & \multicolumn{3}{c|}{ Configuration 3 } \\
\hline $\begin{array}{c}\text { Vehicle } \\
\text { Location }\end{array}$ & $\begin{array}{c}\text { Peak } \\
\text { Event } \\
\text { Duration } \\
(\mathrm{sec})\end{array}$ & $\begin{array}{c}\text { MPE } \\
\text { Margin }\end{array}$ & $\begin{array}{c}\text { MPE } \\
+3 \mathrm{~dB} \\
\text { Margin }\end{array}$ & $\begin{array}{c}\text { Peak } \\
\text { Event } \\
\text { Duration } \\
(\mathrm{sec})\end{array}$ & $\begin{array}{c}\text { MPE } \\
\text { Margin }\end{array}$ & $\begin{array}{c}\text { MPE } \\
+3 \mathrm{~dB} \\
\text { Margin }\end{array}$ & $\begin{array}{c}\text { Peak } \\
\text { Event } \\
\text { Duration } \\
(\mathrm{sec})\end{array}$ & $\begin{array}{c}\text { MPE } \\
\text { Margin }\end{array}$ & $\begin{array}{c}\text { MPE } \\
+3 \mathrm{~dB} \\
\text { Margin }\end{array}$ \\
\hline 1 & 230 & 28.0 & 112 & 10 & 4.1 & 16.3 & 250 & 5.7 & 23.5 \\
\hline 2 & 230 & 19.2 & 77 & 50 & 6.2 & 24.7 & 30 & 10.0 & 40.0 \\
\hline 3 & 10 & 1.0 & 4.0 & 40 & 3.5 & 13.8 & 40 & 28.0 & 112.0 \\
\hline 4 & 40 & 3.9 & 15.8 & 40 & 7.9 & 31.4 & 10 & 5.0 & 20.0 \\
\hline 5 & 40 & 4.7 & 18.7 & 10 & 2.8 & 11.1 & 40 & 21.7 & 86.6 \\
\hline 6 & 40 & 5.6 & 22.2 & \multicolumn{3}{|c|}{ for Peak Event Duration) / (Fatigue Due to Flight Data) } \\
\hline
\end{tabular}

Table 2. Comparison of Expected Peak Loads as Computed From ELV Flight Data, the Associated MPE, and the Associated MPE $+3 \mathrm{~dB}$ based on a comparison of the shock response spectra in Figures 1-3(b).

\begin{tabular}{|c|c|c|c|c|c|c|c|c|c|}
\hline & \multicolumn{2}{|c|}{ Configuration 1 } & \multicolumn{3}{c|}{ Configuration 2 } & \multicolumn{3}{c|}{ Configuration 3 } \\
\hline $\begin{array}{c}\text { Vehicle } \\
\text { Location }\end{array}$ & $\begin{array}{c}\text { Peak } \\
\text { Event } \\
\text { Duration } \\
(\mathrm{sec})\end{array}$ & $\begin{array}{c}\text { MPE } \\
\text { Margin }\end{array}$ & $\begin{array}{c}\text { MPE } \\
+3 \mathrm{~dB} \\
\text { Margin }\end{array}$ & $\begin{array}{c}\text { Peak } \\
\text { Event } \\
\text { Duration } \\
(\mathrm{sec})\end{array}$ & $\begin{array}{c}\text { MPE } \\
\text { Margin }\end{array}$ & $\begin{array}{c}\text { MPE } \\
+3 \mathrm{~dB} \\
\text { Margin }\end{array}$ & $\begin{array}{c}\text { Peak } \\
\text { Event } \\
\text { Duration } \\
(\mathrm{sec})\end{array}$ & $\begin{array}{c}\text { MPE } \\
\text { Margin }\end{array}$ & $\begin{array}{c}\text { MPE } \\
+3 \mathrm{~dB} \\
\text { Margin }\end{array}$ \\
\hline 1 & 230 & 1.9 & 2.7 & 10 & 1.1 & 1.5 & 250 & 1.4 & 1.9 \\
\hline 2 & 230 & 2.0 & 2.8 & 50 & 1.1 & 1.6 & 30 & 1.2 & 1.7 \\
\hline 3 & 10 & 1.4 & 1.9 & 40 & 1.3 & 1.8 & 40 & 1.5 & 2.1 \\
\hline 4 & 40 & 1.3 & 1.8 & 40 & 1.3 & 1.8 & 10 & 1.3 & 1.9 \\
\hline 5 & 40 & 1.1 & 1.6 & 10 & 1.3 & 1.8 & 40 & 1.1 & 1.6 \\
\hline 6 & 40 & 1.3 & 1.9 & & & & & & \\
\hline
\end{tabular}

Notes: MPE Margin = (Peak Load Due to MPE for Peak Event Duration) / (Peak Load Due to Flight Data) $\mathrm{MPE}+3 \mathrm{~dB}$ Margin $=($ Peak Load Due to MPE+3dB for Peak Event Duration $) /($ Peak Load Due to Flight Data $)$ 